\title{
Invisible nanowires with interfering electric and toroidal dipoles
}

\author{
Wei Liu, ${ }^{1, *}$ Jianfa Zhang, ${ }^{1}$ Bing Lei, ${ }^{1}$ Haojun Hu, ${ }^{1}$ and Andrey E. Miroshnichenko ${ }^{2}$ \\ ${ }^{1}$ College of Optoelectronic Science and Engineering, National University of Defense Technology, \\ 137 Yanwachi, Changsha, Hunan 410073, China \\ ${ }^{2}$ Nonlinear Physics Centre, Australian National University, Acton, ACT 0200, Australia \\ *Corresponding author: wei.liu.pku@gmail.com
}

Received February 27, 2015; revised April 21, 2015; accepted April 22, 2015;

posted April 23, 2015 (Doc. ID 235349); published May 8, 2015

\begin{abstract}
By studying the scattering of normally incident plane waves by a single nanowire, we reveal the indispensable role of toroidal multipole excitation in multipole expansions of radiating sources. It is found that for both $p$-polarized and $s$-polarized incident waves, toroidal dipoles can be effectively excited within homogenous dielectric nanowires in the optical spectrum regime. We further demonstrate that the plasmonic core-shell nanowires can be rendered invisible through destructive interference of the electric and toroidal dipoles, which may inspire many nanowire-based light-matter interaction studies, and incubate biological and medical applications that require noninvasive detections and measurements. (C) 2015 Optical Society of America

OCIS codes: (290.5839) Scattering, invisibility; (240.6680) Surface plasmons; (260.5740) Resonance.

http://dx.doi.org/10.1364/OL.40.002293
\end{abstract}

In the conventional Cartesian multipole expansion method, the radiation sources have been decomposed into electric and magnetic multipoles, where the contributions of toroidal components are usually overlooked [1]. In contrast to electric and magnetic counterparts, the toroidal multipole (TM) can be viewed as poloidal current configurations, which flow on the surface of a torus along its meridians, or as a series of magnetic dipoles (enclosed circulating currents) aligned along an enclosed path, which interacts only with the time derivatives of the incident fields $[2,3]$. Though it is long known that the conventional Cartesian multipole expansion method is incomplete as the indispensable role played by TMs is not discussed [2,3], TMs have only attracted significant attention since the recent experimental demonstration of the toroidal responses of metamaterials consisting of specially arranged split ring resonators [4]]. Following this work, shortly afterward in various other structures and spectrum regimes the excitation of TMs have also been identified, which are expected to be of fundamental importance for various applications including biosensing, nanoantennas, photovoltaic devices, and so on [ $\underline{5}-\underline{11}]$.

In currently flourishing fields of plasmonics and metamaterials, the scattering by individual particles plays a foundational role [12-16]. Nevertheless, most demonstrations of the indispensable contributions of TMs have been conducted in engineered structures consisting of relatively complex nanoparticle dimers or clusters [5-11]. Only recently such investigations have been extended to individual nanodisks and nanospheres, which reveal that TMs cannot only be effectively excited but also are essential for accurate interpretations for some fundamental scattering properties $[\underline{17}, \underline{18}]$. In contrast to spherical structures, cylindrical structures show strong polarization dependence, with the nature and relative magnitudes of the modes excited being contrastingly different [19]. To introduce TMs into the scattering nanowires may give novel and complete explanations for some exotic nanowire scattering properties, and thus further inspire extra nanowire-based applications and offer new platforms for studies of strong light-matter interactions. Such investigations into individual nanowires are vitally important, and have not been discussed so far.

In this Letter, we conduct an analytical study on the scattering of plane waves by nanowires with TMs incorporated. It is found that toroidal dipoles (TDs) can be effectively excited in homogenous dielectric nanowires for both $p$-polarized and $s$-polarized incident waves in the optical regime. We further show that in core-shell plasmonic nanowires, the electric dipoles (EDs) and TDs excited can interfere destructively in the far field, thus rendering the nanowires invisible. It is expected that our new TM-based interpretations of the nanowire scattering could possibly incubate many extra applications based on nanowires and inspire the establishment of new platforms for studies into strong light-matter interactions.

In Fig. 1 we show schematically the structure under consideration. The coordinate system on top shows the configuration of the incident plane wave: it is $s$-polarized $(E$ field along nanowire axis $z$ ) and $p$-polarized ( $E$ field normal to the nanowire axis $z$ ) for the nanowires in Figs. 1(a) and 1(b), respectively, both of which are

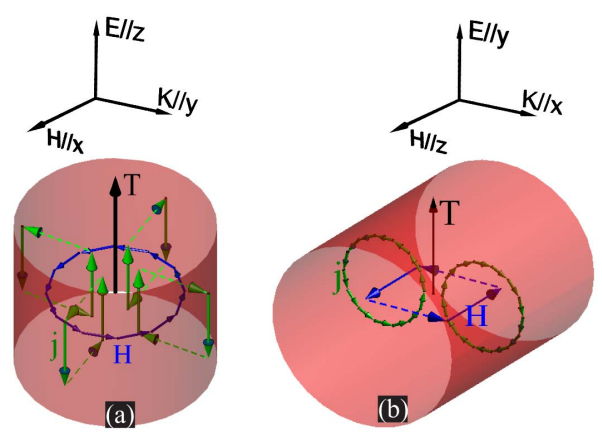

Fig. 1. Schematic of the nanowire scatterings of normally incident (a) $s$-polarized and (b) $p$-polarized plane waves. The TMs excited within the nanowires are illustrated through the current j [or electric field $\mathbf{E}$ according to Eq. (2)] and magnetic field $\mathbf{H}$ distributions. 

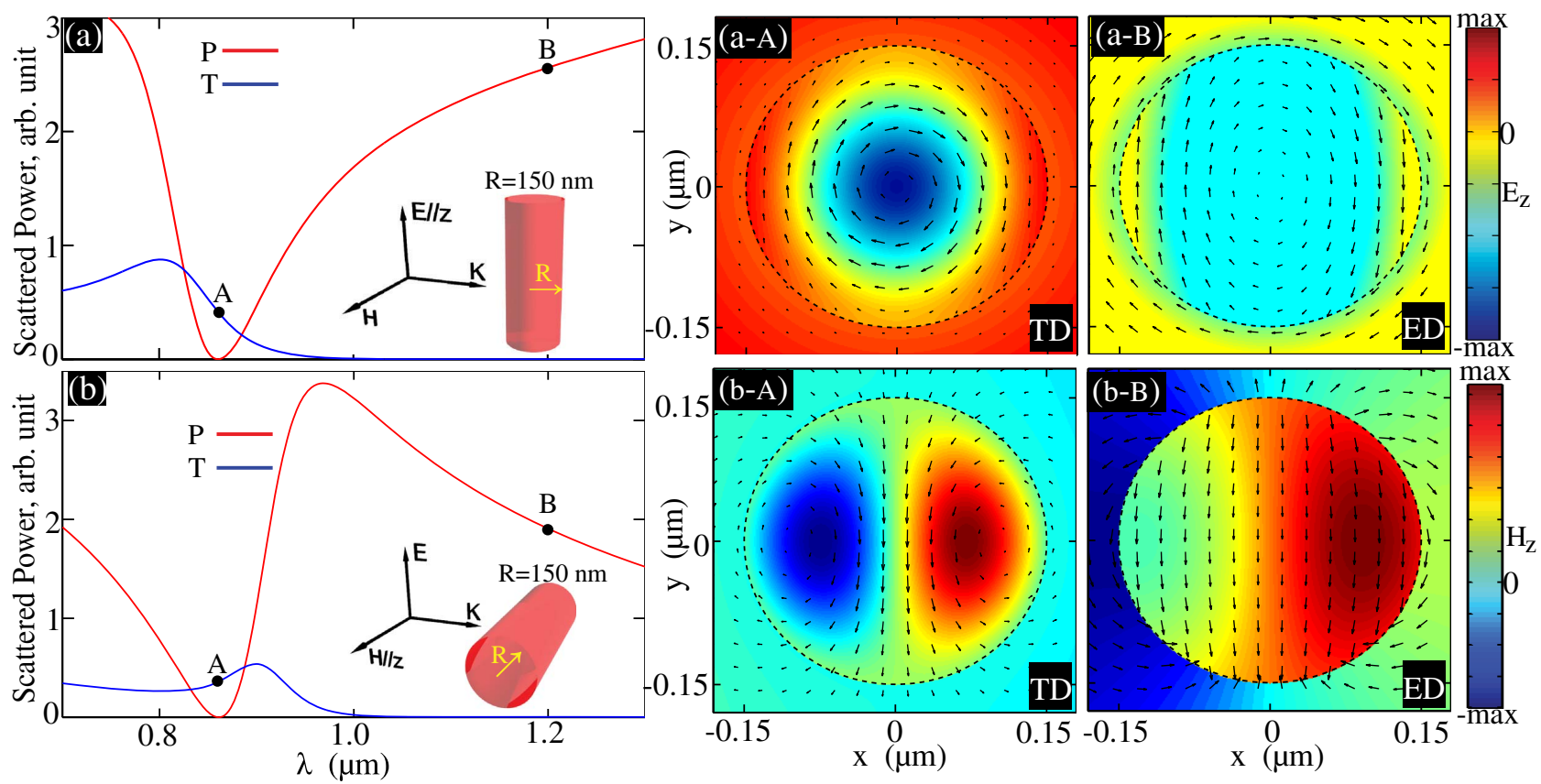

Fig. 2. Scattered power spectra for a dielectric $(n=3.5)$ nanowire of radius $R=150 \mathrm{~nm}$ for (a) $s$-polarized and (b) $p$-polarized plane waves. The scattering configurations are shown as insets in (a) and (b), respectively. For both polarizations, the contributions from current-integrated [based on Eq. (1)] ED (P, red curves) and TD (T, blue curves) are shown. Two points A $(\lambda=860 \mathrm{~nm})$ and B $(\lambda=1200 \mathrm{~nm})$ are marked in (a) and (b), and the corresponding near fields are shown in (a-A) and (a-B) for $s$-polarized incident wave [the color plots and vector plots correspond to longitudinal electric field $\left(E_{z}\right)$ and transverse magnetic field, respectively] and in $(\mathrm{b}-\mathrm{A})$ and $(\mathrm{b}-\mathrm{B})$ for $p$-polarized incident wave [the color plots and vector plots correspond to longitudinal magnetic field $\left(H_{z}\right)$ and transverse electric field, respectively].

assumed to be infinitely long. According to the complete multipole expansion method that has included TMs, the EDs and TDs excited within the nanowires can be expressed by the integrations of the near-field currents [we use the notation of the $\exp (-i \omega t)$ for electromagnetic waves] [묘, $\underline{8}$ :

$$
\mathbf{P}=\frac{1}{-i \omega} \int \mathrm{d}^{2} r \mathbf{j}, \quad \mathbf{T}=\frac{1}{10 c} \int \mathrm{d}^{2} r\left[(\mathbf{r} \cdot \mathbf{j}) \mathbf{r}-2 r^{2} \mathbf{j}\right],
$$

where the displacement current $\mathbf{j}$ is

$$
\mathbf{j}=-i \omega \epsilon_{0}[\epsilon(\mathbf{r})-1] \mathbf{E}(\mathbf{r}),
$$

$\epsilon_{0}$ is the vacuum permittivity, $\epsilon(\mathbf{r})$ is the relative permittivity of the nanowire, $\omega$ is the angular frequency of the incident wave, and $c$ is the speed of light. For both spherical and cylindrical structures, there are analytical expressions for the fields inside [12] and thus both $\mathrm{P}$ and $\mathbf{T}$ can be calculated directly.

In Figs. 1(a) and 1(b) we also show schematically the magnetic field $\mathbf{H}$ and current $\mathbf{j}$ [or electric field $\mathbf{E}$ according to Eq. (2)] distributions of the TDs excited within the nanowires for both polarizations. As is shown, the TD can be simply interpreted as a series of magnetic dipoles (enclosed circulating currents) aligned along an enclosed path, which actually also represents the field-lines of the induced magnetic field $\mathbf{H}$. The scattering power of ED and TD in Eq. (1) on the $x-y$ plane are respectively: $\frac{k^{3} c}{8 \epsilon_{0}} \mathbf{P}^{2}$ and $\frac{k^{5} c}{8 \epsilon_{0}} \mathbf{T}^{2}$ for $s$-polarization; $\frac{k^{3} c}{16 \epsilon_{0}} \mathbf{P}^{2}$ and $\frac{k^{5} c}{16 \epsilon_{0}} \mathbf{T}^{2}$ for $p$-polarization. The scattered power spectra of the EDs and TDs excited [calculated according to Eq. (1) within homogenous dielectric (refractive index $n=3.5$ ) nanowire of radius $R=150 \mathrm{~nm}$ ] with incident waves of both polarizations are shown in Figs. 2(a) and 2(b). It is clear that the TDs can be effectively excited for both $s$ - and $p$-polarized waves [see the blue curves in Figs. 2(a) and 2(b), respectively]. To further verify this, we have selected two points A and B in the scattering power spectra $(\lambda=860 \mathrm{~nm}$ and $\lambda=1200 \mathrm{~nm}$, respectively) and show the near-field distributions at those points in Figs. 2(a-A) to 2(b-B). According to Figs. 2(a) and 2(b), at point $\mathrm{A}$ the excitation of $\mathrm{ED}$ is negligible and thus there is only TD excitation (modes of magnetic type and of higher orders have not been considered here [14, 19-21]). For $s$-polarization the color plots and vector plots correspond to longitudinal electric field $\left(E_{z}\right)$ and transverse magnetic field, respectively [see Figs. 2(a-A) and 2(a-B)]; while for $p$-polarization the color plots and vector plots correspond to longitudinal magnetic field $\left(H_{z}\right)$ and transverse electric field, respectively [see Figs. 2(b-A) and 2(b-B)].

For s-polarization: (1) At point A, according to Fig. 2(a-A), within the nanowire region $E_{z}$ [and thus $\mathbf{j}$ according to Eq. (2)] changes the direction (there are both positive and negative values) and the magnetic field has been mostly confined to the nanowire. This exactly corresponds to current-field distribution for the TD shown in Fig. 1(a). (2) At point B, however, since there is only $\mathrm{ED}$ excitation, neither $E_{z}$ changes its sign nor the magnetic field has been confined within the nanowire [see Fig. 2(a-B)], which corresponds to a typical ED field distribution. For $p$-polarization: (1) At point A, clearly 

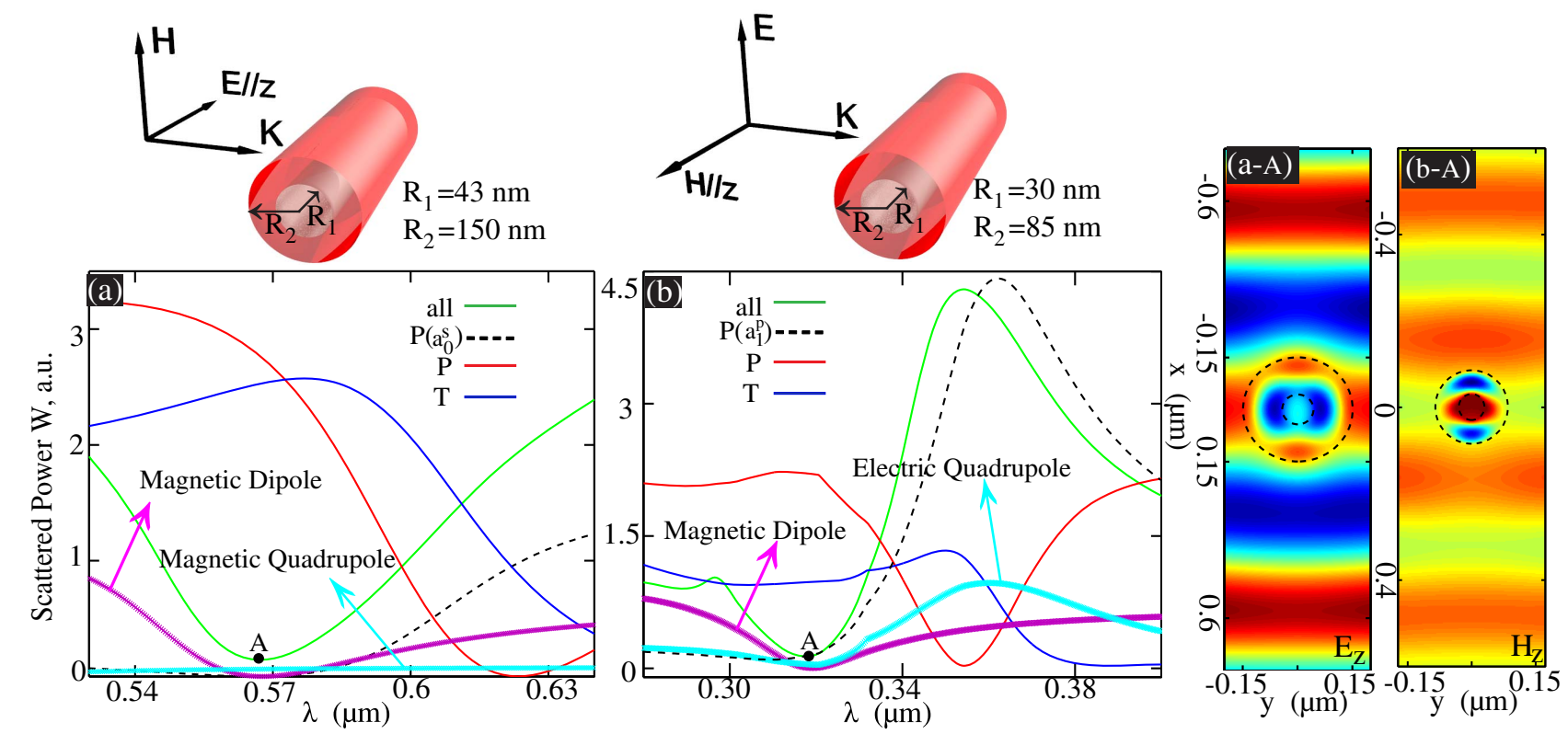

Fig. 3. Scattered power spectra for silver core-dielectric $(n=3.5)$ shell nanowires of inner radius $R_{1}$ and outer radius $R_{2}$ are shown in (a) $R_{1}=43 \mathrm{~nm}$ and $R_{2}=150 \mathrm{~nm}$ of $s$-polarization and (b) $R_{1}=30 \mathrm{~nm}$ and $R_{2}=85 \mathrm{~nm}$ of $p$-polarization [the curves in (b) are not smooth due to the uneven permittivity data of Ag]. The specific scattering configurations are also included above (a) and (b), respectively. For both polarizations the total scattered power [all, green curves, see Eq. (3)], and the contributions from far-field deduced ED in Eq. (4) [dashed black curves, $\mathbf{P}\left(a_{0}^{s}\right)$ for $s$-polarization and $\mathbf{P}\left(a_{1}^{p}\right)$ for $p$-polarization], current-integrated ED (P, red curves) and TD (T, blue curves) are shown. For clarity, we have also provided the contributions from far-field deduced magnetic dipoles and quadrupoles (magnetic and electric types for $s$ and $p$ polarizations, respectively). The transparency points A have been marked $\left[\lambda=566 \mathrm{~nm}\right.$ in (a) and $\lambda=319 \mathrm{~nm}$ in (b)] and the corresponding near-field distributions are shown in (a-A) in terms of $E_{z}$ and (b-A) in terms of $H_{z}$, respectively. The wave is propagating from negative $x$ to positive $x$ direction.

there are both clockwise and anti-clockwise current flows and the magnetic field has been mostly confined within the nanowire [see Fig. 2(b-A)], which corresponds to a typical current-field distribution of the TD [can be compared to Fig. 1(b)] [17,18]. (2) In contrast, at point $\mathrm{B}$, the field distribution is clearly of a ED type [see Fig. 2(b-B)]. We note that in sharp contrast to the demonstration of TD excitation in nanowire clusters for only $s$-polarization [11], our work shows in even individual nanowires, TD can be effectively excited for both polarizations where the cluster configuration is not required.

So far we have studied ED and TD obtained through current integration and the scattering by nanowires (single or multilayered) can be solved analytically and the total scattered power of all multipoles is [12]

$$
W_{\mathrm{sca}}^{s, p}=2 \epsilon_{0} \omega^{-1}\left|E_{0} c\right|^{2}\left[\left(a_{0}^{s, p}\right)^{2}+2 \sum_{m=1}^{\infty}\left(a_{m}^{s, p}\right)^{2}\right],
$$

where $k=2 \pi / \lambda$ is the angular wavenumber in the background material (vacuum in this Letter); $a_{0}$ and $a_{m}$ are the spherical scattering coefficients, where the superscripts $s$ and $p$ correspond to $s$-polarized and $p$-polarized incident waves, respectively. Moreover the scattered fields of the nanowires can be also expressed analytically in terms of $a_{0}$ and $a_{m}$. When compared to the far-field scattering of standard electric and magnetic multipoles, the EDs excited with the nanowires for $s$-polarization and $p$-polarization can be deduced, respectively as [는 21$]$

$$
\mathbf{P}\left(a_{0}^{s}\right)=\epsilon_{0} \frac{4 a_{0}^{s}}{i k^{2}} \mathbf{E}_{0}, \quad \mathbf{P}\left(a_{1}^{p}\right)=\epsilon_{0} \frac{8 a_{1}^{p}}{i k^{2}} \mathbf{E}_{0},
$$

where $\mathbf{E}_{0}$ is the electric field of the incident wave. Thus the scattered power from $\mathbf{P}\left(a_{0}^{s}\right)$ and $\mathbf{P}\left(a_{1}^{p}\right)$ is $2 \omega^{-1} \epsilon_{0}\left|a_{0}^{s} E_{0} c\right|^{2}$ and $4 \omega^{-1} \epsilon_{0}\left|a_{1}^{p} E_{0} c\right|^{2}$, respectively. We note here that in the far field, the ED and TD are indistinguishable since they have identical scattering patterns [2,3]. The above deduced EDs [see Eq. (4), which corresponds to the overall dipolar scattering] have actually combined the contributions from both the EDs (P) and TDs (T) [see Eq. (1)], and some other higher-order (in terms of $k$ ) terms (such as the mean-square radius of TD) obtained through current integration $[\underline{8}, \underline{11}, \underline{17}, \underline{18}]$. As a result, when ED and TD are tuned to appropriate magnitudes and thus can cancel the scattering of each other, invisible scattering bodies can be obtained $[\underline{8}, \underline{11}, \underline{17}, \underline{18}]$.

To demonstrate the invisibility induce by the interferences of EDs and TDs excited within nanowires, we focus on the widely studied core-shell plasmonic nanowires. The analytical results are shown in Fig. 3. The structures under consideration are the silver core-dielectric $(n=$ 3.5) shell nanowires of inner layer radius $R_{1}$ and outer layer radius $R_{2}$ [see the scattering configurations above Figs. 3(a) and 3(b)]. For the permittivity of silver we adopt the experimental data from [22]. For $s$-polarization, $R_{1}=$ $43 \mathrm{~nm}$ and $R_{2}=150 \mathrm{~nm}$ and the scattered power spectra are shown in Fig. 3(a). We have included the contributions from ED (P) and TD (T) (red and blue curves, respectively), the deduced ED [dashed black curve, $\mathbf{P}\left(a_{0}^{s}\right)$ ], and the total scattered power from all multipoles [green curve, see Eq. (3)]. At point A $(\lambda=566 \mathrm{~nm}) \mathrm{ED}$ and TD scatter almost the same amount of power and between them there is complete destructive interference, thus leading to more or less null dipolar scattering at this point 
[see the dashed black curve for $\mathbf{P}\left(a_{0}^{s}\right)$ ]. Moreover, at this point all the other multipole excitations (magnetic dipole and quadrupole, for example) have been significantly suppressed, and consequently the total scattering at this point is negligible [see the green curve in Fig. 3(a)]. For $p$-polarization with $R_{1}=30 \mathrm{~nm}$ and $R_{2}=85 \mathrm{~nm}$, similar effects can be observed in Fig. 3(b). The difference is that the minimum scattering point $\overline{\mathrm{A}(\lambda}=319 \mathrm{~nm})$ is not the point $(\lambda \approx 340 \mathrm{~nm})$ where ED and TD scatter equally and between them there is complete destructive interference. This is due to the fact that for the overall dipolar scattering [dashed black curve Fig. 3(b)], besides the destructive-interfered scattering from TD and ED, there are also non-negligible contributions from the mean-square radius of the ED and TD terms and some other higher-order terms [3].

To further verify the induced invisibility, we present near-field distributions at the points marked in Figs. 3(a) and 3(b) in Fig. 3(a-A) ( $E_{z}$, for $s$-polarization) and Fig. 3(b-A) ( $H_{z}$ for $s$-polarization), respectively. It is clear that the incident waves have experienced almost no perturbations for both polarizations, verifying the invisibility of the nanowires. Moreover, there are still significant field distributions inside the nanowires at those invisible points, which agrees with the results shown in Figs. 3(a) and 3(b) that there are both ED and TD excitation inside. To summarize, we have demonstrated invisible coreshell nanowires originating from interferences of EDs and TDs excited for both polarizations. Though there have been lots of studies on the transparency phenomena of core-shell nanowires (see [23-25], for example), unfortunately none of them have noticed the roles played by TMs. We note that the invisibility we demonstrate is basically a resonant effect and cannot be achieved over a spectral regime of broad bandwidth.

To conclude, we study the scattering of normally incident plane waves by nanowires through a complete Cartesian multipole expansion method where TMs have been considered. It is shown that for both $p$-polarized and $s$-polarized incident waves, TDs can be effectively excited within homogenous dielectric nanowires. We further demonstrate the TD-induced invisibility in coreshell plasmonic nanowires, which simultaneously have significant internal field distribution and negligible scattering (signal can be received but not disturbed), and thus might play a significant role in noninvasive detections and measurements. Here we confine our studies to TDs and such investigations can certainly be extended to other higher-order TMs. The incorporation of TMs into scattering nanowires may offer new insights into the understanding of many nanowire-based light matter interactions, and hopefully can inspire lots of applications of nanowires in sensing, nanoantennas, lasers, photovoltaic devices, and so on.

We acknowledge the financial support from the National Natural Science Foundation of China (Grant
Nos: 11404403, 11304389, and 61205141), and the Australian Research Council (FT110100037).

\section{References}

1. J. D. Jackson, Classical Electrodynamics (Wiley, 1962).

2. V. M. Dubovik and V. V. Tugushev, Phys. Rep. 187, 145 (1990).

3. E. E. Radescu and G. Vaman, Phys. Rev. E 65, 046609 (2002).

4. T. Kaelberer, V. Fedotov, N. Papasimakis, D. Tsai, and N. Zheludev, Science 330, 1510 (2010).

5. Z. Dong, P. Ni, J. Zhu, X. Yin, and X. Zhang, Opt. Express 20, 13065 (2012).

6. Y. W. Huang, Opt. Express 20, 1760 (2012).

7. B. Ogut, N. Talebi, R. Vogelgesang, W. Sigle, and P. A. van Aken, Nano Lett. 12, 5239 (2012).

8. V. A. Fedotov, A. Rogacheva, V. Savinov, D. Tsai, and N. I. Zheludev, Sci. Rep. 3, 2967 (2013).

9. Y. Fan, Z. Wei, H. Li, H. Chen, and C. M. Soukoulis, Phys. Rev. B 87, 115417 (2013).

10. V. Savinov, V. Fedotov, and N. Zheludev, Phys. Rev. B 89, 205112 (2014).

11. A. A. Basharin, M. Kafesaki, E. N. Economou, C. M. Soukoulis, V. A. Fedotov, V. Savinov, and N. I. Zheludev, Phys. Rev. X 5, 011036 (2015).

12. C. F. Bohren and D. R. Huffman, Absorption and Scattering of Light by Small Particles (Wiley, 1983).

13. W. Liu, A. E. Miroshnichenko, D. N. Neshev, and Y. S. Kivshar, ACS Nano 6, 5489 (2012).

14. W. Liu, A. E. Miroshnichenko, and Y. S. Kivshar, Chin. Phys. B 23, 047806 (2014).

15. S. Campione, L. I. Basilio, L. K. Warne, and M. B. Sinclair, Opt. Express 23, 2293 (2015).

16. T. Coenen, F. Bernal Arango, A. Femius Koenderink, and A. Polman, Nat. Commun. 5, 3250 (2014).

17. A. E. Miroshnichenko, A. B. Evlyukhin, Y. F. Yu, R. M. Bakker, A. Chipouline, A. I. Kuznetsov, B. Lukyanchuk, B. N. Chichkov, and Y. S. Kivshar, "Seeing the unseen: observation of an anapole with dielectric nanoparticles," arXiv:1412.0299 (2014).

18. W. Liu, J. Zhang, and A. E. Miroshnichenko, "Toroidal dipole induced transparency for core-shell nanoparticles," arXiv:1412.4931 (2014).

19. W. Liu, A. E. Miroshnichenko, R. F. Oulton, D. N. Neshev, O. Hess, and Y. S. Kivshar, Opt. Lett. 38, 2621 (2013).

20. K. Vynck, D. Felbacq, E. Centeno, A. I. Cabuz, D. Cassagne, and B. Guizal, Phys. Rev. Lett. 102, 133901 (2009).

21. E. Kallos, I. Chremmos, and V. Yannopapas, Phys. Rev. B 86, 245108 (2012).

22. P. B. Johnson and R. W. Christy, Phys. Rev. B 6, 4370 (1972).

23. Z. Ruan and S. Fan, J. Phys. Chem. C 114, 7324 (2010).

24. P. Fan, U. K. Chettiar, L. Cao, F. Afshinmanesh, N. Engheta, and M. L. Brongersma, Nat. Photonics 6, 380 (2012).

25. A. Mirzaei, I. V. Shadrivov, A. E. Miroshnichenko, and Y. S. Kivshar, Opt. Express 21, 10454 (2013). 